\title{
Uniqueness results for nonlinear fractional differential equations with infinite-point integral boundary conditions
}

\author{
Suli Liua , Junpeng Liuª , Qun Dai ${ }^{b}$, Huilai Li ${ }^{a}{ }^{a} *$ \\ ${ }^{a}$ Department of Mathematics, Jilin University, Changchun, 130012, P. R. China. \\ ${ }^{b}$ College of Science, Changchun University of Science and Technology, Changchun, 130022, P. R. China.
}

\author{
Communicated by $\mathrm{Y}$. Hu
}

\begin{abstract}
In this paper, we consider a class of nonlinear fractional differential equations involving the Riemann-Liouville fractional derivative with infinite-point integral boundary conditions. Our analysis relies on the fixed point index theory and $u_{0}$-positive operator. An example is given for the illustration of the main work. (C)2017 All rights reserved.
\end{abstract}

Keywords: Fractional differential equations, infinite-point integral boundary condition, $\mathfrak{u}_{0}$-positive operator, fixed point index theory.

2010 MSC: 26A33, 34A34.

\section{Introduction}

Fractional calculus developed since 17th century through the pioneering works of Leibniz, Euler, Lagrange, Liouville and many other researchers and has been investigated in diverse. The recent development covers the theoretical as well as potential applications of the subject in physical and technical science. Recently, Atangana and Baleanu proposed a derivative with fractional order based upon the Mittag-Leffler function which has a non-singular and nonlocal kernel, see [2,3] and the references therein. Fractional differential equations have been of great interest recently, see $[6,10,11]$. There are many results dealing with the existence and multiplicity of solutions of nonlinear fractional differential equations by the means of techniques of nonlinear analysis, see $[1,4,8,9,12]$.

In [13], Zhang studied the existence of positive solutions of the following nonlinear fractional differential equations with infinite-point boundary value conditions:

$$
\left\{\begin{array}{l}
D_{0^{+}}^{\alpha} u(t)+q(t) f(t, u(t))=0, \quad 0<t<1, n-1<\alpha \leqslant n, \\
u(0)=u^{\prime}(0)=\cdots=u^{(n-2)}(0)=0, u^{(i)}(1)=\sum_{j=1}^{\infty} \alpha_{j} u\left(\xi_{j}\right),
\end{array}\right.
$$

\footnotetext{
*Corresponding author

Email addresses: liusl15@mails.jlu.edu.cn (Suli Liu), liujp14@mails.jlu.edu.cn (Junpeng Liu), daiqun1130@163.com (Qun Dai), lihuilai@jlu.edu.cn (Huilai Li)

doi:10.22436/jnsa.010.03.37
} 
where $\alpha>2, i \in[1, n-2]$ is a fixed integer, $\alpha_{j} \geqslant 0,0<\xi_{1}<\xi_{2}<\cdots<\xi_{j-1}<\xi_{j}<\cdots<1(j=$ $1,2, \ldots), \Delta-\sum_{j=1}^{\infty} \alpha_{j} \xi_{j}^{\alpha-1}>0, \Delta=(\alpha-1)(\alpha-2) \cdots(\alpha-i)$, and $D_{0^{+}}^{\alpha}$ is the standard Riemann-Liouville derivative. By using the fixed point theorem, several local existence and multiplicity of positive solutions were obtained.

Motivated by the work mentioned above, in this paper, we study the following nonlinear fractional differential equations with infinite-point integral boundary value conditions:

$$
\left\{\begin{array}{l}
D_{0^{+}}^{\alpha} u(t)+q(t) f(t, u(t))=0, \quad 0<t<1, n-1<\alpha \leqslant n, \\
u(0)=u^{\prime}(0)=\cdots=u^{(n-2)}(0)=0, u^{(i)}(1)=\sum_{j=1}^{\infty} \alpha_{j} I_{0^{+}}^{\beta} u\left(\xi_{j}\right),
\end{array}\right.
$$

where $\alpha>2, \beta \geqslant 0, i \in[0, n-2]$ is a fixed integer, $\alpha_{j} \geqslant 0,0<\xi_{1}<\xi_{2}<\cdots<\xi_{j-1}<\xi_{j}<\cdots<1(j=$ $1,2, \ldots), \Delta-\sum_{j=1}^{\infty} \frac{\Gamma(\alpha)}{\Gamma(\alpha+\beta)} \alpha_{j} \xi_{j}^{\alpha+\beta-1}>0$ ( $\Delta$ will be defined in Lemma 2.3 below), and $D_{0^{+}}^{\alpha}$ is the standard Riemann-Liouville differential operator.

Here, we emphasize that the infinite-point integral boundary value condition of (1.1) can be understood in the sense that the value of the unknown function $u^{(i)}(t)$ at the position $t=1$ is proportional to the infinite-point Riemann-Liouville integral of the unknown function $\sum_{j=1}^{\infty} \alpha_{j} I_{0^{+}}^{\beta} u\left(\xi_{j}\right)$. Furthermore, for $\beta=0$, the integral boundary value condition of (1.1) reduces to the usual form of infinite-point boundary value condition $u^{(i)}(1)=\sum_{j=1}^{\infty} \alpha_{j} u\left(\xi_{j}\right)$, which has been considered in [13].

The rest of the paper is organized as follows. In Section 2, we list some lemmas. The uniqueness results are given in Section 3. In Section 4, an example is presented to illustrate the main results.

\section{Preliminaries}

We list the following assumptions adopted in this paper:

$\left(A_{1}\right) \mathrm{q}:(0,1) \rightarrow[0,+\infty)$ is continuous and $0<\int_{0}^{1} \mathrm{p}(\mathrm{s}) \mathrm{d} s<+\infty$;

$\left(A_{2}\right) f:[0,1] \times \mathbb{R} \rightarrow \mathbb{R}$ is continuous.

For the convenience of the reader, we present here some necessary definitions of the fractional calculus which can be found in the recent literature $[6,10,11]$.

Definition 2.1. The Riemann-Liouville fractional integral of order $\alpha>0$ of a function $f:(0, \infty) \rightarrow \mathbb{R}$ is given by

$$
\mathrm{I}_{0^{+}}^{\alpha} \mathrm{f}(\mathrm{t})=\frac{1}{\Gamma(\alpha)} \int_{0}^{\mathrm{t}}(\mathrm{t}-\mathrm{s})^{\alpha-1} \mathrm{f}(\mathrm{s}) \mathrm{d} \mathrm{s}
$$

provided that the right-hand side is pointwise defined on $(0, \infty)$.

Definition 2.2. The Riemann-Liouville fractional derivative of order $\alpha>0$ of a continuous function $f:(0, \infty) \rightarrow \mathbb{R}$ is given by

$$
D_{0^{+}}^{\alpha} f(t)=\frac{1}{\Gamma(n-\alpha)}\left(\frac{d}{d t}\right)^{n} \int_{0}^{t}(t-s)^{n-\alpha-1} f(s) d s
$$

where $n-1 \leqslant \alpha<n$, provided that the right-hand side is pointwise defined on $(0, \infty)$.

Lemma 2.3. Assume that $\mathrm{y}(\mathrm{t}) \in \mathrm{C}([0,1])$, then the problem

$$
\left\{\begin{array}{l}
D_{0^{+}}^{\alpha} u(t)+y(t)=0, \quad 0<t<1, n-1<\alpha \leqslant n, \\
u(0)=u^{\prime}(0)=\cdots=u^{(n-2)}(0)=0, u^{(i)}(1)=\sum_{j=1}^{\infty} \alpha_{j} I_{0^{+}}^{\beta} u\left(\xi_{j}\right),
\end{array}\right.
$$


where $\Delta-\sum_{j=1}^{\infty} \frac{\Gamma(\alpha)}{\Gamma(\alpha+\beta)} \alpha_{j} \xi_{j}^{\alpha+\beta-1}>0, i \in[0, n-2]$ is a fixed integer, is equivalent to

$$
u(t)=\int_{0}^{1} G(t, s) y(s) d s
$$

where

$$
G(t, s)=\frac{1}{p(0) \Gamma(\alpha)} \begin{cases}t^{\alpha-1} p(s)(1-s)^{\alpha-1-i}-p(0)(t-s)^{\alpha-1}, & 0 \leqslant s \leqslant t \leqslant 1 \\ t^{\alpha-1} p(s)(1-s)^{\alpha-1-i,} & 0 \leqslant t \leqslant s \leqslant 1\end{cases}
$$

with $p(s):=\Delta-\Sigma_{s \leqslant \xi_{j}} \frac{\Gamma(\alpha)}{\Gamma(\alpha+\beta)} \alpha_{j}\left(\xi_{j}-s\right)^{\alpha+\beta-1}(1-s)^{i+1-\alpha}$ and

$$
\Delta= \begin{cases}1, & i=0 \\ (\alpha-1)(\alpha-2) \cdots(\alpha-i), & i \geqslant 1\end{cases}
$$

$\mathrm{G}(\mathrm{t}, \mathrm{s})$ is called Green's function of FBVP $(2.1)$. Obviously, $\mathrm{G}(\mathrm{t}, \mathrm{s})$ is a continuous function on $[0,1] \times[0,1]$.

Proof. It is easy to see that problem (2.1) is equivalent to the following integral equation

$$
u(t)=-I_{0^{+}}^{\alpha} y(t)+C_{1} t^{\alpha-1}+C_{2} t^{\alpha-2}+\cdots+C_{n} t^{\alpha-n} .
$$

By $u(0)=u^{\prime}(0)=\cdots=u^{(n-2)}(0)=0$, we have

$$
\mathrm{C}_{2}=\mathrm{C}_{3}=\cdots=\mathrm{C}_{\mathrm{n}}=0 .
$$

Then, we get

$$
u(t)=-I_{0^{+}}^{\alpha} y(t)+C_{1} t^{\alpha-1}
$$

$\operatorname{By~} u^{(i)}(1)=\sum_{j=1}^{\infty} \alpha_{j} I_{0^{+}}^{\beta} u\left(\xi_{j}\right)$, we have

$$
-\mathrm{I}_{0^{+}}^{\alpha-i} \mathrm{y}(1)+\mathrm{C}_{1} \Delta=\sum_{j=1}^{\infty} \alpha_{j}\left\{-\mathrm{I}_{0^{+}}^{\alpha+\beta} y\left(\xi_{j}\right)+\mathrm{C}_{1} \frac{\Gamma(\alpha)}{\Gamma(\alpha+\beta)} \xi_{j}^{\alpha+\beta-1}\right\} .
$$

When $\Delta-\sum_{j=1}^{\infty} \frac{\Gamma(\alpha)}{\Gamma(\alpha+\beta)} \alpha_{j} \xi_{j}^{\alpha+\beta-1} \neq 0$, we obtain that

$$
C_{1}=\frac{1}{\Delta-\sum_{j=1}^{\infty} \frac{\Gamma(\alpha)}{\Gamma(\alpha+\beta)} \alpha_{j} \xi_{j}^{\alpha+\beta-1}}\left\{I_{0^{+}}^{\alpha-i} y(1)-\sum_{j=1}^{\infty} \alpha_{j} I_{0^{+}}^{\alpha+\beta} y\left(\xi_{j}\right)\right\} .
$$

Let $p(s):=\Delta-\Sigma_{s \leqslant \xi_{j}} \frac{\Gamma(\alpha)}{\Gamma(\alpha+\beta)} \alpha_{j}\left(\xi_{j}-s\right)^{\alpha+\beta-1}(1-s)^{i+1-\alpha}$, then $p(0)=\Delta-\sum_{j=1}^{\infty} \frac{\Gamma(\alpha)}{\Gamma(\alpha+\beta)} \alpha_{j} \xi_{j}^{\alpha+\beta-1}$. Therefore, the solution to problem (2.1) is

$$
\begin{aligned}
u(t)=- & I_{0^{+}}^{\alpha} y(t)+\frac{t^{\alpha-1}}{p(0)}\left\{I_{0^{+}}^{\alpha-i} y(1)-\sum_{j=1}^{\infty} \alpha_{j} I_{0^{+}}^{\alpha+\beta} y\left(\xi_{j}\right)\right\} \\
=- & \frac{1}{\Gamma(\alpha)} \int_{0}^{t}(t-s)^{\alpha-1} y(s) d s \\
& +\frac{t^{\alpha-1}}{p(0)}\left\{\frac{1}{\Gamma(\alpha-i)} \int_{0}^{1}(1-s)^{\alpha-i-1} y(s) d s-\sum_{j=1}^{\infty} \frac{\alpha_{j}}{\Gamma(\alpha+\beta)} \int_{0}^{\xi_{j}}\left(\xi_{j}-s\right)^{\alpha+\beta-1} y(s) d s\right\} \\
= & \frac{1}{p(0) \Gamma(\alpha)}\left\{-p(0) \int_{0}^{t}(t-s)^{\alpha-1} y(s) d s+\Delta t^{\alpha-1}\left\{\int_{0}^{t}+\int_{t}^{1}\right\}(1-s)^{\alpha-i-1} y(s) d s\right.
\end{aligned}
$$




$$
\begin{aligned}
& \left.-\sum_{j=1}^{\infty} \frac{\alpha_{j} \Gamma(\alpha)}{\Gamma(\alpha+\beta)} t^{\alpha-1} \int_{0}^{\xi_{j}}\left(\xi_{j}-s\right)^{\alpha+\beta-1} y(s) d s\right\} \\
= & \int_{0}^{1} G(t, s) y(s) d s .
\end{aligned}
$$

The proof is finished.

Lemma 2.4. Suppose that $p(0)>0$, then $p(s)>0, s \in[0,1]$, and $p(s)$ is nondecreasing on $[0,1]$.

Proof. By simple computation, we know that $p^{\prime}(s)>0$ which implies that $p(s)$ is nondecreasing on $[0,1]$. We omit the details.

It is clear that $p(s)-p(0)>0, \forall s \in(0,1]$, and $\frac{p(s)-p(0)}{s}$ is continuous for all $s \in(0,1]$. Then, there exists $M_{1} \geqslant m_{1}>0$, such that $m_{1} s+p(0) \leqslant p(s) \leqslant M_{1} s+p(0)$, where

$$
m_{1}:=\inf _{0<s \leqslant 1} \frac{p(s)-p(0)}{s}, M_{1}:=\sup _{0<s \leqslant 1} \frac{p(s)-p(0)}{s} .
$$

Lemma 2.5. The function $\mathrm{G}(\mathrm{t}, \mathrm{s})$ defined by (2.2) satisfies the following conditions:

(i) $\mathrm{p}(0) \Gamma(\alpha) \mathrm{G}(\mathrm{t}, \mathrm{s}) \geqslant \mathrm{m}_{1} \mathrm{~s}(1-\mathrm{s})^{\alpha-1-\mathrm{i}_{\mathrm{t}}} \mathrm{t}^{\alpha-1}, \forall \mathrm{t}, \mathrm{s} \in[0,1]$;

(ii) $p(0) \Gamma(\alpha) G(t, s) \leqslant\left[M_{1}+p(0)\right](1-s)^{\alpha-1-i} t^{\alpha-1}, \forall t, s \in[0,1]$;

(iii) $\mathrm{G}(\mathrm{t}, \mathrm{s})>0, \forall \mathrm{t}, \mathrm{s} \in(0,1)$.

Proof. For $0 \leqslant s \leqslant t \leqslant 1$, we have

$$
\begin{aligned}
p(0) \Gamma(\alpha) G(t, s) & =t^{\alpha-1} p(s)(1-s)^{\alpha-1-i}-p(0)(t-s)^{\alpha-1} \\
& =[p(s)-p(0)](1-s)^{\alpha-1-i} t^{\alpha-1}+p(0)\left[(1-s)^{\alpha-1-i} t^{\alpha-1}-(t-s)^{\alpha-1}\right] \\
& \geqslant m_{1} s(1-s)^{\alpha-1-i} t^{\alpha-1}+p(0)\left[(1-s)^{\alpha-1} t^{\alpha-1}-(t-s)^{\alpha-1}\right] \\
& \geqslant m_{1} s(1-s)^{\alpha-1-i} t^{\alpha-1}
\end{aligned}
$$

and

$$
\begin{aligned}
p(0) \Gamma(\alpha) G(t, s) & =t^{\alpha-1} p(s)(1-s)^{\alpha-1-i}-p(0)(t-s)^{\alpha-1} \\
& =[p(s)-p(0)](1-s)^{\alpha-1-i} t^{\alpha-1}+p(0)\left[(1-s)^{\alpha-1-i} t^{\alpha-1}-(t-s)^{\alpha-1}\right] \\
& \leqslant M_{1} s(1-s)^{\alpha-1-i} t^{\alpha-1}+p(0)(1-s)^{\alpha-1-i_{t}} t^{\alpha-1} \\
& \leqslant\left[M_{1}+p(0)\right](1-s)^{\alpha-1-i} t^{\alpha-1} .
\end{aligned}
$$

For $0 \leqslant \mathrm{t} \leqslant \mathrm{s} \leqslant 1$, we have

$$
p(0) \Gamma(\alpha) G(t, s)=t^{\alpha-1} p(s)(1-s)^{\alpha-1-i} \geqslant[p(s)-p(0)](1-s)^{\alpha-1-i_{t}} t^{\alpha-1} \geqslant m_{1} s(1-s)^{\alpha-1-i} t^{\alpha-1},
$$

and

$$
\begin{aligned}
p(0) \Gamma(\alpha) G(t, s) & =t^{\alpha-1} p(s)(1-s)^{\alpha-1-i} \\
& =[p(s)-p(0)](1-s)^{\alpha-1-i} t^{\alpha-1}+p(0)(1-s)^{\alpha-1-i} t^{\alpha-1} \\
& \leqslant M_{1} s(1-s)^{\alpha-1-i} t^{\alpha-1}+p(0)(1-s)^{\alpha-1-i} t^{\alpha-1} \\
& \leqslant\left[M_{1}+p(0)\right](1-s)^{\alpha-1-i} t^{\alpha-1} .
\end{aligned}
$$

The proof is finished. 
Let Banach space $E=C([0,1])$ be endowed with the norm $\|u\|_{\infty}=\max _{0 \leqslant t \leqslant 1}|u(t)|$, and $\theta$ is the zero function in $E$. Define a closed cone $P \subset E$ by $P=\{u \in E \mid u(t) \geqslant 0, t \in[0,1]\}$.

Lemma 2.6 (Krein-Rutmann theorem [5]). Suppose that $\mathrm{T}: \mathrm{E} \rightarrow \mathrm{E}$ is a completely continuous linear operator and $\mathrm{T}(\mathrm{P}) \subseteq \mathrm{P}$. If there exist $\psi \in \mathrm{C}[0,1] \backslash(-\mathrm{P})$ and a constant $\mathrm{c}>0$ such that $\mathrm{c} T \psi \geqslant \psi$, then the spectral radius $r(T) \neq 0$ and $T$ has a positive eigenfunction $\varphi_{1}$ corresponding to its first eigenvalue $\lambda_{1}=(r(T))^{-1}$.

Definition 2.7 ([7]). We say that a bounded linear operator $T: E \rightarrow E$ is $u_{0}$-positive on the cone $P$ if there exists $u_{0} \in P \backslash\{\theta\}$ such that for every $x \in P \backslash\{\theta\}$ there exist a natural number $n$ and positive functions $\alpha(x)>0, \beta(x)>0$ such that

$$
\alpha(x) u_{0} \leqslant T^{n} x \leqslant \beta(x) u_{0},
$$

where $\theta$ is the zero function in $E$. Furthermore, if $u_{0}=\varphi_{1}$, the positive eigenfunction of $T$ corresponding to its first eigenvalue $\lambda_{1}$, then $T$ is a $\varphi_{1}$-positive operator.

Define the operator $A: E \rightarrow E$ by

$$
(A u)(t)=\int_{0}^{1} G(t, s) q(s) f(s, u(s)) d s, t \in[0,1] .
$$

It is not hard to see that the fixed points of operator A coincide with the solutions to the FBVP (1.1).

Define the operator $T: E \rightarrow E$ by

$$
(T u)(t)=\int_{0}^{1} G(t, s) q(s) u(s) d s, t \in[0,1] .
$$

It is not difficult to verify that $\mathrm{T}: \mathrm{P} \rightarrow \mathrm{P}$ is a completely continuous linear operator. By virtue of the Krein-Rutmann theorem, we have the following lemma.

Lemma 2.8. Suppose $\mathrm{T}$ is defined by (2.3), then the spectral radius $\mathrm{r}(\mathrm{T}) \neq 0$ and $\mathrm{T}$ has a positive eigenfunction $\varphi_{1}$ corresponding to its first eigenvalue $\lambda_{1}=(\mathrm{r}(\mathrm{T}))^{-1}$.

Proof. By Lemma 2.5, $G(t, s)>0$ for all $t, s \in(0,1)$. Take $\left[t_{1}, t_{2}\right] \subset(0,1), q(t)>0, \forall t \in\left[t_{1}, t_{2}\right]$, choose $\psi \in E$ such that $\psi(t) \geqslant 0$ for all $t \in[0,1], \psi(t)>0$ for all $t \in\left[t_{1}, t_{2}\right], \psi(t)=0$ for all $t \in\left[0, t_{1}\right) \cup\left(t_{2}, 1\right]$. Thus, we have

$$
(T \psi)(t)=\int_{0}^{1} G(t, s) q(s) \psi(s) d s=\int_{t_{1}}^{t_{2}} G(t, s) q(s) \psi(s) d s>0 .
$$

So, there exists a constant $c>0$ such that $c(T \psi)(t) \geqslant \psi(t), \forall t \in[0,1]$. By Lemma 2.6, we complete the proof.

Lemma 2.9. $T$ is $\mathrm{u}_{0}$-positive operator with $\mathrm{u}_{0}(\mathrm{t})=\mathrm{t}^{\alpha-1}$. In addition, $\mathrm{T}$ is $\varphi_{1}$-positive operator, where $\varphi_{1}$ is the positive eigenfunction corresponding to its first eigenvalue.

Proof. For any $x \in P \backslash\{\theta\}$, by Lemma 2.5, we have

$$
(T x)(t)=\int_{0}^{1} G(t, s) q(s) x(s) d s \leqslant \frac{1}{p(0) \Gamma(\alpha)} \int_{0}^{1}\left[M_{1}+p(0)\right](1-s)^{\alpha-1-i} q(s) x(s) d s \cdot t^{\alpha-1} .
$$

On the other hand, we have

$$
(T x)(t)=\int_{0}^{1} G(t, s) q(s) x(s) d s \geqslant \frac{1}{p(0) \Gamma(\alpha)} \int_{0}^{1} m_{1} s(1-s)^{\alpha-1-i} q(s) x(s) d s \cdot t^{\alpha-1} .
$$

Therefore, $T$ is $u_{0}$-positive operator with $u_{0}(t)=t^{\alpha-1}$, i.e.,

$$
\alpha(x) u_{0} \leqslant T x \leqslant \beta(x) u_{0}, \quad \forall x \in P \backslash\{\theta\} .
$$


Let $\varphi_{1}$ be the positive eigenfunction of $T$ corresponding to $\lambda_{1}$, i.e., $\varphi_{1}=\lambda_{1} T \varphi_{1}$. Then, there exist $\tilde{\alpha}\left(\varphi_{1}\right), \tilde{\beta}\left(\varphi_{1}\right)>0$ such that

$$
\tilde{\alpha}\left(\varphi_{1}\right) u_{0} \leqslant T \varphi_{1}=\frac{1}{\lambda_{1}} \varphi_{1} \leqslant \tilde{\beta}\left(\varphi_{1}\right) u_{0} .
$$

Hence, we obtain that $T$ is $\varphi_{1}$-positive operator. The proof is completed.

\section{Main results}

Theorem 3.1. Suppose that there exists $\mathrm{K} \in\left[0, \lambda_{1}\right)$ such that

$$
|f(t, u)-f(t, v)| \leqslant K|u-v|, \forall u, v \in E,
$$

where $\lambda_{1}$ is the first eigenvalue of $\mathrm{T}$. Then the FBVP (1.1) has a unique solution $\mathrm{u}^{*}$ in $\mathrm{E}$, moreover, for any $\mathrm{u}_{0} \in \mathrm{E}$, there exists iterative sequence $\left\{u_{\mathfrak{n}}\right\}_{\mathfrak{n}=0}^{\infty}$ with

$$
u_{n+1}=A u_{n}, \lim _{n \rightarrow \infty} u_{n}=u^{*}, n=0,1,2, \ldots
$$

Proof. Firstly, it is not hard to see that the fixed points of operator A coincide with the solutions to the problem (1.1).

Secondly, we will show that $A$ has fixed points in $E$. For any given $u_{0} \in E$, let $u_{n+1}=A u_{n}$. By Lemma 2.9 , there exists $\beta=\beta\left(\left|u_{1}-u_{0}\right|\right)>0$, such that

$$
\left(T\left|u_{1}-u_{0}\right|\right)(t) \leqslant \beta \varphi_{1}(t), t \in[0,1] .
$$

Let $K=k \lambda_{1}, k \in[0,1)$, for any $m \in \mathbb{N}$, we have

$$
\begin{aligned}
\left|u_{m+1}-u_{m}\right| & =\left|\left(A u_{m}\right)(t)-\left(A u_{m-1}\right)(t)\right| \\
& =\left|\int_{0}^{1} G(t, s) q(s)\left[f\left(s, u_{m}(s)\right)-f\left(s, u_{m-1}(s)\right)\right] d s\right| \\
& \leqslant \int_{0}^{1} G(t, s) q(s)\left|f\left(s, u_{m}(s)\right)-f\left(s, u_{m-1}(s)\right)\right| d s \\
& \leqslant k \lambda_{1} \int_{0}^{1} G(t, s) q(s)\left|u_{m}-u_{m-1}\right| d s \\
& =k \lambda_{1} T\left(\left|u_{m}-u_{m-1}\right|\right)(t) \\
& \leqslant \cdots \leqslant k^{m} \lambda_{1}^{m} T^{m}\left(\left|u_{1}-u_{0}\right|\right)(t) \\
& \leqslant k^{m} \lambda_{1}^{m} T^{m-1} \beta \varphi_{1}(t) \\
& =\beta k^{m} \lambda_{1}^{m-1} T^{m-2} \varphi_{1}(t)=\beta k^{m} \lambda_{1} \varphi_{1}(t) .
\end{aligned}
$$

Thus, for $n, m \in \mathbb{N}$, we have

$$
\begin{aligned}
\left|u_{n+m+1}-u_{n}\right| & =\left|u_{n+m+1}-u_{n+m}+\cdots+u_{n+1}-u_{n}\right| \\
& \leqslant\left|u_{n+m+1}-u_{n+m}\right|+\cdots+\left|u_{n+1}-u_{n}\right| \\
& \leqslant \beta\left[k^{n+m}+\cdots+k^{n}\right] \lambda_{1} \varphi_{1}(t) \\
& =\beta \lambda_{1} \frac{k^{n}\left(1-k^{m+1}\right)}{1-k} \varphi_{1}(t) .
\end{aligned}
$$

Therefore,

$$
0 \leqslant\left\|u_{n+m+1}-u_{n}\right\| \leqslant \beta \lambda_{1} \frac{k^{n}\left(1-k^{m+1}\right)}{1-k}\left\|\varphi_{1}(t)\right\| \rightarrow 0, \quad \text { as } n, m \rightarrow \infty .
$$


By the completeness of $E$, there exists a $u^{*} \in E$ such that $\lim _{n \rightarrow \infty} u_{n}=u^{*}$.

Thus, $u^{*}=\lim _{n \rightarrow \infty} u_{n+1}=\lim _{n \rightarrow \infty} A u_{n}=A u^{*}, A$ have fixed points in $E$.

Finally, we will show that $A$ has at most one fixed point in $E$. Suppose there exist two fixed points $u, v \in E, u=A u, v=A v$. By Lemma 2.9, there exists $\beta=\beta(|u-v|)>0$, such that

$$
(T|u-v|)(t) \leqslant \beta \varphi_{1}(t), \quad t \in[0,1] .
$$

Then for all $n \in \mathbb{N}$, the following holds

$$
|u-v|=\left|A^{n} u-A^{n} v\right| \leqslant \beta k^{n} \lambda_{1} \varphi_{1} .
$$

This means that $u=v$, and $A$ has at most one fixed point in $E$. The proof is completed.

Remark 3.2. The iterative sequence in Theorem 3.1 starting with a simple function is helpful for calculating.

By the same method of [4], we have the following results. We omit the details.

Theorem 3.3. Suppose that there exist $\mathrm{u}_{0} \in \mathrm{E}, \mathrm{K} \in\left[0, \lambda_{1}\right)$ such that

$$
\left\{\begin{array}{l}
D_{0^{+}}^{\alpha} u_{0}(t)+q(t) f\left(t, u_{0}(t)\right) \geqslant 0, \quad 0<t<1, n-1<\alpha \leqslant n, \\
u_{0}(0)=u_{0}^{\prime}(0)=\cdots=u_{0}^{(n-2)}(0)=0, u_{0}^{(i)}(1) \leqslant \sum_{j=1}^{\infty} \alpha_{j} I_{0^{+}}^{\beta} u_{0}\left(\xi_{j}\right), \\
0 \leqslant f(t, u)-f(t, v) \leqslant k(u-v), u(t) \geqslant v(t), \forall t \in[0,1], u, v \in \Omega,
\end{array}\right.
$$

where $\lambda_{1}$ is the first eigenvalue of $\mathrm{T}, \Omega=\left\{\mathrm{u} \in \mathrm{E} \mid \mathrm{u} \geqslant \mathrm{u}_{0}\right\}$. Then the FBVP (1.1) has a unique solution $\mathrm{u}^{*}$ in $\Omega$.

Theorem 3.4. Suppose that there exist $\mathrm{u}_{0} \in \mathrm{E}, \mathrm{K} \in\left[0, \lambda_{1}\right)$ such that

$$
\left\{\begin{array}{l}
D_{0^{+}}^{\alpha} u_{0}(t)+q(t) f\left(t, u_{0}(t)\right) \leqslant 0, \quad 0<t<1, n-1<\alpha \leqslant n, \\
u_{0}(0)=u_{0}^{\prime}(0)=\cdots=u_{0}^{(n-2)}(0)=0, u_{0}^{(i)}(1) \geqslant \sum_{j=1}^{\infty} \alpha_{j} I_{0^{+}}^{\beta} u_{0}\left(\xi_{j}\right), \\
0 \leqslant f(t, u)-f(t, v) \leqslant K(u-v), \quad u(t) \geqslant v(t), \forall t \in[0,1], u, v \in \Omega,
\end{array}\right.
$$

where $\lambda_{1}$ is the first eigenvalue of $\mathrm{T}, \Omega=\left\{\mathrm{u} \in \mathrm{E} \mid \mathrm{u} \leqslant \mathrm{u}_{0}\right\}$. Then the FBVP (1.1) has a unique solution $\mathrm{u}^{*}$ in $\Omega$.

\section{An example}

Example 4.1. Consider the following problem

$$
\left\{\begin{array}{l}
D_{0^{+}}^{7 / 2} u(t)+\lambda_{0}(1-t)^{-1 / 2}\left[\frac{1}{3} u+2+5 t^{3}+\sin t+\frac{1}{2} t^{2} u\right]=0,0<t<1, \\
u(0)=u^{\prime}(0)=u^{\prime \prime}(0)=0, u^{\prime}(1)=\sum_{j=1}^{\infty} \frac{8}{j^{2}} I_{0+}^{3 / 2} u\left(\frac{1}{2 j}\right),
\end{array}\right.
$$

where $\alpha=\frac{7}{2}, i=1, \alpha_{j}=\frac{8}{j^{2}}, \beta=\frac{3}{2}, \xi_{j}=\frac{1}{2 j}, \Gamma\left(\frac{7}{2}\right) \approx 3.32335, \Delta=2.5, p(0)=\Delta-\sum_{j=1}^{\infty} \frac{\Gamma(\alpha)}{\Gamma(\alpha+\beta)} \alpha_{j} \xi_{j}^{\alpha+\beta-1} \approx$ $2.38611>0$, and $q(t)=(1-t)^{-1 / 2}, f(t, u(t))=\lambda_{0}\left[\frac{1}{3} u+2+5 t^{3}+\sin t+\frac{1}{2} t^{2} u\right], 0<\lambda_{0}<\lambda_{1}, \lambda_{1}$ is the first eigenvalue of the operator $\mathrm{T}$.

It is clear that $\left(\mathrm{A}_{1}\right),\left(\mathrm{A}_{2}\right)$ hold. For all $u, v \in E$, we have

$$
|f(t, u(t))-f(t, v(t))| \leqslant \lambda_{0}\left|\left[\frac{1}{3}(u-v)+\frac{1}{2} t^{2}(u-v)\right]\right| \leqslant \lambda_{1} \frac{5}{6}|u-v| .
$$

It follows from Theorem 3.1 that FBVP (4.1) has a unique solution, moreover, for any $u_{0} \in E$, there exists iterative sequence $\left\{u_{n}\right\}_{n=0}^{\infty}$ with

$$
u_{n+1}=A u_{n}, \lim _{n \rightarrow \infty} u_{n}=u^{*}, n=0,1,2, \ldots
$$




\section{Acknowledgment}

This work is supported by the National Nature Science Foundation of P. R. China (No. 11271154).

\section{References}

[1] B. Ahmad, R. P. Agarwal, Some new versions of fractional boundary value problems with slit-strips conditions, Bound. Value Probl., 2014 (2014), 12 pages. 1

[2] A. Atangana, D. Baleanu, New fractional derivatives with nonlocal and non-singular kernel: Theory and application to heat transfer model, Therm. Sci., 20 (2016), 763-769. 1

[3] A. Atangana, I. Koca, Chaos in a simple nonlinear system with Atangana-Baleanu derivatives with fractional order, Chaos Solitons Fractals, 89 (2016), 447-454. 1

[4] Y.-J. Cui, Uniqueness of solution for boundary value problems for fractional differential equations, Appl. Math. Lett., 51 (2016), 48-54. 1, 3

[5] D.-J. Guo, Nonlinear integral equations, Shandong Science and Technology Press, Jinan, (1987). 2.6

[6] A. A. Kilbas, H. M. Srivastava, J. J. Trujillo, Theory and applications of fractional differential equations, North-Holland Mathematics Studies, Elsevier Science B.V., Amsterdam, (2006). 1, 2

[7] M. A. Krasnoselskilu, Positive solutions of operator equations, Translated from the Russian by Richard E. Flaherty; edited by Leo F. Boron P. Noordhoff Ltd. Groningen, (1964), 381 pages. 2.7

[8] S.-L. Liu, H.-L. Li, Q. Dai, Nonlinear fractional differential equations with nonlocal integral boundary conditions, Adv. Difference Equ., 2015 (2015), 11 pages. 1

[9] S.-L. Liu, H.-L. Li, Q. Dai, J.-P. Liu, Existence and uniqueness results for nonlocal integral boundary value problems for fractional differential equations, Adv. Difference Equ., 2016 (2016), 14 pages. 1

[10] J. Sabatier, O. P. Agrawal, J. A. Tenreiro Machado (Eds.), Advances in fractional calculus, Theoretical developments and applications in physics and engineering, Including papers from the Minisymposium on Fractional Derivatives and their Applications (ENOC-2005) held in Eindhoven, August 2005, and the 2nd Symposium on Fractional Derivatives and their Applications (ASME-DETC 2005) held in Long Beach, CA, September 2005, Springer, Dordrecht, (2007). 1, 2

[11] S. G. Samko, A. A. Kilbas, O. I. Marichev, Fractional integrals and derivatives, Theory and applications, Edited and with a foreword by S. M. Nikolskiü, Translated from the 1987 Russian original, Revised by the authors, Gordon and Breach Science Publishers, Yverdon, (1993). 1, 2

[12] W.-Z. Xie, J. Xiao, Z.-G. Luo, Existence of extremal solutions for nonlinear fractional differential equation with nonlinear boundary conditions, Appl. Math. Lett., 41 (2015), 46-51. 1

[13] X.-Q. Zhang, Positive solutions for a class of singular fractional differential equation with infinite-point boundary value conditions, Appl. Math. Lett., 39 (2015), 22-27. 1, 1 